\title{
Doença crônica da valva mitral em cães: avaliação clínica funcional e mensuração ecocardiográfica da valva mitral
}

\author{
[Chronic mitral valvular disease in dogs: assessment of functional clinical stage and echocardiographic \\ measurement of the mitral valve] \\ R.A.L. Muzzi ${ }^{1}$, L.A.L. $M u z z i^{1}$, R.B. Araújo $^{2}$, D.A. Lázaro $^{3}$ \\ ${ }^{1}$ Departamento de Medicina Veterinária - UFLA \\ Caixa postal 3037 \\ 37200-000 - Lavras, MG \\ ${ }^{2}$ Escola de Veterinária - UFMG - Belo Horizonte, MG \\ ${ }^{3}$ Aluna de pós-graduação - EE-UFMG - Belo Horizonte, MG
}

\begin{abstract}
RESUMO
Foram descritos os achados do exame físico em cães com degeneração mixomatosa crônica da valva mitral e mensurado o comprimento e a espessura das cúspides da valva mitral pelo exame ecocardiográfico. Utilizaram-se 81 cães de diferentes raças de pequeno a médio porte, com idade de 11,12 $\pm 2,51$ anos e peso de $6,24 \mathrm{~kg} \pm 3,19$, dos quais 20 eram clinicamente normais (grupo-controle) e 61 apresentavam evidências clínicas de cardiopatia. Os animais foram distribuídos em três classes distintas segundo a insuficiência cardíaca congestiva (grupos I, II e III), de acordo com os dados do histórico e dos sinais clínicos apresentados. Ao exame ecocardiográfico, avaliaram-se as medidas das cúspides da valva mitral, que não apresentaram diferença entre os cães do grupo I e II, embora estivessem anormais se comparadas às dos cães do grupocontrole. Nos animais do grupo III, as medidas das valvas avaliadas foram maiores que as dos cães dos grupos I e II. Foram observados nódulos com formato arredondado e, nos cães do grupo III, as cúspides estavam bastante alongadas e espessadas.
\end{abstract}

Palavras-chave: cão, exame ecocardiográfico, valva mitral, coração

\begin{abstract}
Physical and echocardiographic aspects were studied in dogs with chronic mitral valvular disease. The length and thickness of the mitral valve leaflets were echocardiographically measured. Eighty-one dogs of small-medium breeds (mean of $6.24 \mathrm{~kg} \pm 3.19)$, older than six years (11.12 \pm 2.51 years) were used. Twenty were clinically normal dogs (control group) and 61 presented myxomatous degeneration of the mitral valve. The diseased dogs were categorized into three classes of congestive heart failure (I, II, and III groups) according to the information on clinical manifestations. On the echocardiographic exam, the measurements of the mitral valve leaflets were evaluated and no diferences between dogs of groups I and II were found, though they were abnormal if compared with control group animals. Group III dogs presented measurements higher than those of animals of groups I and II, as well as leaflets very thick and round-shaped nodules were observed.
\end{abstract}

Keywords: dog, echocardiographic exam, mitral valve, heart

\section{INTRODUÇÃO}

Entre as cardiopatias valvares adquiridas em cães, a degeneração mixomatosa crônica da valva mitral (DMVM) é a de maior prevalência

Recebido em 18 de setembro de 2007

Aceito em 8 de abril de 2009

E-mail: ralmuzzi@ufla.br

Apoio: FAPEMIG
(Bright e Mears, 1997). Há relatos de que a degeneração valvular acomete a valva mitral isoladamente em $62 \%$ dos casos, podendo ocorrer, ainda, a associação desta com a valva tricúspide em 33\% deles (Atkins, 1995). A prevalência e a gravidade das lesões 
degenerativas mixomatosas estão relacionadas à idade e à raça. Cães idosos e de raças de pequeno porte são os mais afetados (Kittleson, 1998; Sisson et al., 1999).

Os sinais clínicos são decorrentes da disfunção do complexo valvar mitral (Bright e Mears, 1997). A tosse é o sinal clínico mais comum, e é a mais citada observação do proprietário. Os episódios de tosse são frequentemente seguidos de dispneia, taquipneia, ortopneia, perda de apetite e letargia (Atkins, 1994; Bright e Mears, 1997; Kittleson, 1998; Muzzi et al., 1999). Outros achados, frequentemente observados nos quadros mais graves, são fadiga, cianose, tempo de perfusão capilar acima de dois segundos e mucosas pálidas (Kittleson, 1998; Sisson et al., 1999). Ao exame físico, o sopro constitui o achado clínico mais precoce, sendo mais facilmente auscultado no ápice cardíaco esquerdo. À auscultação pulmonar, os ruídos podem estar normais ou revelar crepitações difusas, dependendo do estágio da doença (Bright e Mears, 1997; Kittleson, 1998; Pedersen et al., 1999; Sisson et al., 1999; Morais e Pereira, 2001).

A ecocardiografia é um exame complementar muito útil no diagnóstico da DMVM, sendo exequível e não-invasivo. Embora, para a realização do exame ecocardiográfico, a sedação não seja essencial, pode-se utilizá-la para promover relaxamento no paciente e evitar o estresse. Para Stepien et al. (1995), sedativos seguros e efetivos são necessários na maioria dos procedimentos diagnósticos na medicina veterinária, sobretudo na ecocardiografia, por promoverem um estudo de alta qualidade. É recomendada a combinação de maleato de acepromazina e buprenorfina, a qual, além de bem tolerada hemodinamicamente, permite excelente sedação, sem deteriorar a função cardiorrespiratória. Outra vantagem de utilizar o sedativo é a diminuição da frequência cardíaca, que pode estar aumentada pelo próprio mecanismo fisiopatológico da doença ou por situações de estresse, como os exames clínico ou cardiológico, alterando os valores diagnósticos (Jacobs e Mahjoob, 1988; Cape et al., 1993; Häggström et al., 1995).

Segundo Soares et al. (2005), o exame ecocardiográfico permite a avaliação da gravidade da doença mitral e sua classificação em leve, moderada e grave, com base nas características do fluxo regurgitante e do tamanho atrial esquerdo. Para Shah (1994) e Hyun (2005), além dessas características, pacientes com DMVM apresentam espessamento em graus variados da cúspide septal e, em menor grau, da cúspide parietal da valva mitral, que também apresenta boa correlação com o grau de regurgitação mitral, sendo um método útil na classificação de insuficiência cardíaca. Em humanos, o espessamento valvular é um importante marcador prognóstico, refletindo a gravidade da lesão; quanto maior e mais alongada a cúspide, pior o quadro clínico.

Como não há estudos na medicina veterinária que classifiquem a insuficiência cardíaca com base na espessura das cúspides da valva mitral, esta pesquisa foi realizada com os objetivos de descrever os achados do exame físico em cães com DMVM e mensurar a espessura da valva mitral pelo exame ecocardiográfico, avaliando-se a possibilidade de classificar os pacientes como portadores de insuficiência mitral discreta, moderada ou grave, por meio dessas medidas.

\section{MATERIAL E MÉTODOS}

Foram utilizados 81 cães de raças de pequeno e médio porte, com idade de $11,12 \pm 2,51$ anos e peso médio de $6,24 \pm 3,19 \mathrm{~kg}$, dos quais 61 eram portadores de degeneração mixomatosa crônica da valva mitral e 20 eram clinicamente sadios. Dos 61 cães avaliados com DMVM, 42 eram machos e 19 fêmeas. As raças representadas foram: Poodle toy e médio e seus mestiços (19), Pinscher e seus mestiços (14), Cocker Spaniel Inglês e Americano (3), Yorkshire Terrier (4), Terrier Brasileiro (4), Teckel (3) e Pequinês (2) além de cães sem raça definida (12). O grupocontrole constituiu-se de animais clinicamente sadios (7 machos e 13 fêmeas). As raças observadas neste grupo foram: Pinscher e seus mestiços (10), Cocker Spaniel Inglês e Americano (3), Poodle médio (2) e Yorkshire Terrier (1), e cães sem raça definida (4).

Os animais doentes foram selecionados com base nos seguintes critérios: presença de sopro sistólico de regurgitação mitral, exclusão para uso de medicação para o coração (digitálicos, vasodilatadores ou diuréticos), exclusão para qualquer outra doença cardíaca e idade acima de seis anos. Os animais do grupo-controle foram 
selecionados pela ausência de cardiopatia e idade acima de seis anos.

Os cães foram distribuídos em quatro grupos. $\mathrm{O}$ primeiro foi o grupo-controle, e os outros três seguiram as recomendações de classificação de insuficiência cardíaca congestiva (ICC) do Conselho Internacional de Cardiologia em Pequenos Animais (Bonagura et al., 1992), a saber: grupo I, constituído por cães assintomáticos, identificados como portadores de insuficiência mitral leve (22 animais); grupo II, por cães com ICC de discreta a moderada, classificados como portadores de insuficiência mitral moderada (21 animais) e grupo III, por cães com ICC avançada, classificados como portadores de insuficiência mitral grave (18 animais).

Após o exame clínico geral e específico, os animais foram submetidos ao exame eletrocardiográfico (ECG) ${ }^{1}$ nas derivações I, II, III, aVR, aVL, aVF (velocidade de $50 \mathrm{~mm} / \mathrm{s}, 1 \mathrm{~cm}$ $=1 \mathrm{mV})$.

Os cães foram posicionados em decúbito lateral por meio de contenção manual, sendo previamente submetidos à sedação leve com maleato de acepromazina ${ }^{2} \quad(0,03 \mathrm{mg} / \mathrm{kg})$ e buprenorfina $^{3}(0,0075 \mathrm{mg} / \mathrm{kg})$, ambos por via intravenosa. Aguardou-se um período de 10 a 15 minutos, como recomendado por Stepien et al. (1995), para diminuir o estresse e a ansiedade durante o exame. As frequências cardíaca e respiratória foram medidas antes e 10 minutos após a aplicação.

Empregou-se um aparelho de ultra-sonografia ${ }^{4}$, com acompanhamento eletrocardiográfico no monitor, sendo realizadas de 8 a 10 medidas de cada variável em diferentes ciclos cardíacos. Para a realização do modo bidimensional, foram seguidas as recomendações do Colégio Americano de Medicina Interna Veterinária e do Colégio de Cardiologia Veterinária (Thomas et al., 1993). Nesse modo, foram avaliados as câmaras cardíacas, a contratilidade miocárdica, o

${ }^{1}$ Eletrocardiógrafo Ecafix ECG 5 - Ecafix - São Paulo, Brasil.

${ }^{2}$ Acepran 1\% - Univet AS - São Paulo, Brasil.

${ }^{3}$ Temgesic - Schrering Plough - EUA.

${ }^{4}$ Ecocardiógrafo HP Sonos $100 \mathrm{CF}$ - Hewlett Packard EUA. pericárdio e o aspecto morfológico das valvas cardíacas.

$\mathrm{Na}$ posição paraesternal direita longitudinal quatro câmaras foi realizada a medida do tamanho e da espessura das cúspides da valva mitral. O comprimento das cúspides parietal (CCP) e septal (CCS) foi medido da borda até a inserção no anel mitral. A espessura, tanto da cúspide parietal (ECP) quanto da septal (ECS), foi medida no terço médio, ambas realizadas no final da diástole, na onda $\mathrm{Q}$ do $\mathrm{ECG}$, em milímetros.

Todas as variáveis foram submetidas à análise estatística descritiva e à análise de variância, utilizando-se o SAEG (Sistema ..., 1999). Para a comparação entre médias, utilizou-se o teste SNK $(\mathrm{P}<0,05)$.

\section{RESULTADOS}

Ao exame físico, os animais do grupo-controle estavam em bom estado geral, com mucosas e linfonodos normais e auscultação pulmonar e cardíaca também normais. Os do grupo I não apresentavam sinais clínicos de ICC e foram admitidos no serviço de cardiologia por apresentarem sopro cardíaco. Ao exame físico, apresentavam-se em bom estado geral, tempo de perfusão capilar normal, mucosas e linfonodos normais. À auscultação pulmonar, estavam sem alteração e com sopro de regurgitação meso a holossistólico, de graus I/VI a V/VI em ápice esquerdo, com o ponto de máxima intensidade principalmente em foco mitral, que irradiava dorsal, cranial e também para o hemitórax direito. Em alguns animais, detectou-se sopro de regurgitação mesossistólico em ápice direito, de graus III/VI a V/VI $(\mathrm{n}=5)$.

Os cães do grupo II apresentavam histórico de doença cardíaca e sinais de ICC. A principal observação do proprietário era a tosse, especialmente à noite, cansaço em repouso ou após exercícios leves e, em dois casos, presença de ascite. Ao exame físico, 20 cães (95\%) apresentavam-se em estado geral regular, mucosas normais ou cianóticas, linfonodos normais à palpação e tempo de perfusão capilar normal ou discretamente aumentado. A auscultação pulmonar variou de normal à presença de crepitações difusas. Foi auscultado sopro de regurgitação meso a holossistólico de 
graus IV/VI a VI/VI em ápice esquerdo, com o ponto de máxima intensidade em foco mitral, que irradiava dorsal e cranial, assim como para o hemitórax direito. Em sete animais (33\%) foi detectado sopro de regurgitação mesossistólico também em ápice direito, de graus IV/VI a V/VI.

Todos os animais do grupo III apresentavam sinais clínicos graves de ICC. Os proprietários relataram tosse, cansaço excessivo mesmo em repouso, fadiga, ortopneia, perda de peso, anorexia e letargia. Ao exame físico, apresentavam estado geral regular, mucosas pálidas ou cianóticas, tempo de perfusão capilar aumentado em 16 cães (88\%) e taquipneia. Oito animais (44\%) apresentaram ascite e um, desidratação. Observou-se, ainda, a ocorrência de síncope em um animal. À auscultação pulmonar, constataram-se crepitações de leves a intensas, em todo o campo pulmonar. $\mathrm{Na}$ auscultação cardíaca, foi possível observar sopro de regurgitação meso a holossistólico de graus IV/VI a VI/VI em ápice esquerdo, que irradiava dorsal, cranial e para o hemitórax direito. Nove animais $(50 \%)$ possuíam sopro de regurgitação meso a holossistólico em ápice direito, de graus IV/VI a V/VI. Foi ainda detectado ritmo de galope em quatro cães (22\%). Nenhum animal apresentou efusão pericárdica.
O ECG detectou a presença de arritmia sinusal em 77 animais (95\%), tendo sido ainda observada taquicardia sinusal em um cão do grupo II e em dois do grupo III, e fibrilação atrial em um cão do grupo III. Nos 10 minutos iniciais após a sedação, a frequência cardíaca passou de $117 \pm 18$ batimentos por minuto (bpm) para $106 \pm 14$ bpm e a frequência respiratória de $24 \pm 3,1$ movimentos respiratórios por minuto (mrm) para $20 \pm 3 \mathrm{mrm}$.

Os resultados das medidas do comprimento e da espessura das cúspides parietal (CCP e ECP) e septal (CCS e ECS) da valva mitral são apresentados na Tab. 1. Nos cães sadios, a valva mitral não apresentou espessamento nem prolapso valvar. Nos portadores de DMVM, a valva encontrava-se com graus variados de espessamento e prolapso em direção ao átrio esquerdo. Tanto o comprimento quanto a espessura das cúspides apresentaram diferença entre os grupos. Os animais do grupo III demonstraram grau de espessamento grave e os dos grupos I e II, grau de espessamento discreto a moderado. Sete cães, especialmente do grupo III, apresentaram espessamentos localizados na borda livre da cúspide, principalmente na septal.

Tabela 1. Médias e desvios-padrão de medidas ecocardiográficas, modo bidimensional da valva mitral de cães com degeneração mixomatosa crônica da valva mitral (grupos I, II e III) e do grupo-controle

\begin{tabular}{ccccc}
\hline \multirow{2}{*}{ Variável } & \multicolumn{4}{c}{ Medidas ecocardiográficas (em mm) } \\
\cline { 2 - 5 } & Grupo controle & Grupo I & Grupo II & Grupo III \\
\hline CCS & $11,4 \pm 2,27 \mathrm{c}$ & $14,07 \pm 2,97 \mathrm{~b}$ & $14,89 \pm 2,48 \mathrm{~b}$ & $18,15 \pm 2,83 \mathrm{a}$ \\
ECS & $1,17 \pm 0,24 \mathrm{c}$ & $2,55 \pm 0,60 \mathrm{~b}$ & $2,80 \pm 0,62 \mathrm{~b}$ & $3,39 \pm 0,71 \mathrm{a}$ \\
CCP & $7,97 \pm 1,36 \mathrm{c}$ & $9,19 \pm 1,77 \mathrm{~b}, \mathrm{c}$ & $9,85 \pm 2,22 \mathrm{~b}$ & $11,99 \pm 2,91 \mathrm{a}$ \\
ECP & $1,17 \pm 0,23 \mathrm{c}$ & $2,08 \pm 0,49 \mathrm{~b}$ & $2,09 \pm 0,61 \mathrm{~b}$ & $2,54 \pm 0,64 \mathrm{a}$ \\
\hline
\end{tabular}

CCS: comprimento da cúspide septal da valva mitral; ECS: espessura da cúspide septal da valva mitral; CCP: comprimento da cúspide parietal da valva mitral; ECP: espessura da cúspide parietal da valva mitral. Valores seguidos por letras distintas na linha diferem entre si, pelo teste $\operatorname{SNK}(\mathrm{P}<0,05)$.

\section{DISCUSSÃO}

A degeneração mixomatosa crônica da valva mitral representa uma importante cardiopatia adquirida em cães e que leva a sinais aparentes de disfunção e ICC em graus variados (Atkins, 1995), como observado neste estudo. Todos os cardiopatas foram agrupados nas três classes funcionais de ICC, como citado por Bonagura et al. (1992), que elaboraram diretrizes para classificar funcionalmente os animais dependendo dos sinais clínicos e da tolerância ao exercício.

A ocorrência da DMVM relacionou-se à raça e ao sexo, acometendo, principalmente os cães de raças de pequeno porte e machos, aspecto já descrito por Atkins (1995) e Pedersen (2000).

Os animais do grupo I não apresentaram qualquer sinal de ICC, exceto sopro suave, meso a holossistólico, mais audível no ápice esquerdo, de graus III/VI a IV/VI, também descritos por 
Häggström et al. (1995), Bright e Mears (1997) e Morais e Pereira (2001).

Os do grupo II apresentaram sinais de ICC e sopro mais intenso, de grau IV/VI a VI/VI. Em alguns animais foi detectado sopro também no ápice direito. Pedersen et al. (1999), Pedersen (2000) e Soares et al. (2005) relataram que, com a progressão do quadro, o sopro aumenta de intensidade, passando para holossistólico e irradiando-se para o lado direito. Häggström et al. (1995) citaram que esse sopro sistólico está relacionado com a classe de ICC, pois, na doença valvar crônica, ocorre espessamento progressivo e retração das cúspides da valva, aumentando o orifício e, consequentemente, o sopro. Segundo Bright e Mears (1997), é difícil diferenciar a insuficiência tricúspide associada à de mitral de um sopro mitral que irradia para o lado direito. Todos os animais do grupo II apresentaram sinais de ICC, especialmente tosse, citada como a principal observação do proprietário, que ocorria principalmente à noite. Atkins (1994) explica que as causas da tosse podem ser variadas, embora ainda não estejam completamente compreendidas. Pode ser explicada pelo aumento progressivo do $\mathrm{AE}$, que eleva e pressiona o brônquio principal esquerdo, pelo edema da mucosa das vias respiratórias, pela pressão do fluido intersticial nas vias aéreas ou, ainda, pela produção excessiva de muco bronquial. Os sons pulmonares crepitantes, detectados em todos os cães deste grupo, foram de leves a difusos. Bright e Mears (1997) relataram que podem ocorrer variações no grau do edema pulmonar de acordo com o estágio da doença, levando à dispneia e à ortopneia.

Os cães do grupo III apresentaram os sinais mais graves de ICC. Para Bright e Mears (1997) e Muzzi et al. (1999), na doença mitral crônica em grau avançado, os animais podem apresentar perda de peso, intolerância ao exercício, tosse, dispneia, taquipneia e tempo de perfusão capilar aumentado. A ascite é resultante de regurgitação concomitante de tricúspide e ICC direita, que pode representar um achado tardio da regurgitação mitral grave. Kittleson (1998) descreveu a síncope, ocorrida em um animal desse grupo, como consequência de alguma arritmia, vasodilatação aguda ou, ainda, secundária à tosse intensa, comumente chamada de desmaio tussígeno.
As auscultações cardíaca e pulmonar evidenciaram quadro grave em quase todos os animais do grupo III (16/18), sendo o sopro holossistólico de grau VI/VI, em sua maioria, e com intensidade igual em ambos os lados do tórax. O ritmo de galope, constatada em quatro cães desse grupo, já havia sido relatado por Atkins (1995) como indicativo de insuficiência miocárdica avançada na presença de ICC esquerda.

Dos 61 animais portadores de DMVM nenhum apresentou efusão pleural. Segundo Kittleson (1998), isto é de ocorrência incomum e, geralmente, associada à ICC direita, secundária à regurgitação tricúspide.

Ao exame eletrocardiográfico, houve predominância da arritmia sinusal, mas também presença de taquicardia sinusal e fibrilação atrial. O ECG é considerado inespecífico e de pouco valor para o diagnóstico da DMVM (Pedersen, 2000; Soares et al., 2005). Nos quadros mais graves puderam ser detectadas arritmias, como a fibrilação atrial, que Guglielmini et al. (2000) e Soares et al. (2005) citaram estar associadas ao aumento do átrio esquerdo (AE). $\mathrm{O}$ animal que apresentou fibrilação atrial pertencia ao grupo III e apresentava aumento acentuado do AE (área = $12,4 \mathrm{~cm}^{2} /$ normal $\leq 5,0 \mathrm{~cm}^{2}$ ). Para Guglielmini et al. (2000), a baixa prevalência de fibrilação atrial deve-se ao fato de o $\mathrm{AE}$ não atingir o tamanho suficiente para induzir essa arritmia, especialmente quando são acometidos animais de porte tão reduzidos, como os observados neste estudo.

Com o emprego da sedação para a realização do exame ecocardiográfico, utilizando-se buprenorfina e maleato de acepromazina, não foram detectadas alterações significativas nos parâmetros clínicos. Foi observada discreta diminuição da frequência respiratória, que Stepien et al. (1995) descreveram como sendo uma mudança resultante mais da diminuição da ansiedade pela sedação do que pelas alterações ácido-básicas ou efeitos diretos nos centros de controle respiratório.

Nos cães que participaram da pesquisa, a frequência cardíaca também apresentou discreta redução o que, segundo Stepien et al. (1995), pode ocorrer devido ao opióide, que causa aumento no tônus vagal, reduzindo a frequência 
cardíaca. Foi importante o controle principalmente da frequência cardíaca antes do exame ecocardiográfico, pois Jacobs e Mahjoob (1988) relataram que o aumento na frequência cardíaca altera significativamente as dimensões do ventrículo esquerdo (VE), podendo afetar, inclusive, a função ventricular esquerda.

Cape et al. (1993) descreveram que o aumento na frequência cardíaca poderia diminuir o tamanho do jato de regurgitação no Doppler em cores, podendo subestimar sua medida, o que afetaria a credibilidade da variável na avaliação semiquantitativa da gravidade da lesão. Stepien et al. (1995), ao utilizarem até mesmo dosagens maiores que as empregadas nesse estudo, indicaram que a associação da acepromazina com buprenorfina é bem tolerada pelos cães, não causando alterações hemodinâmicas, sendo satisfatória em relação à sedação para procedimentos diagnósticos ou terapêuticos.

Segundo Bright e Mears (1997), Boon (1998) e Soares et al. (2005), o exame ecocardiográfico, modo bidimensional, é muito útil no diagnóstico da DMVM, especialmente na avaliação do aparato valvar e das câmaras cardíacas, sobretudo quando há dilatação do AE. Para Carabello (1998) e Teshima et al. (2007), este exame é indispensável na avaliação do paciente com doença mitral, principalmente no que se refere à análise da gravidade e extensão da regurgitação, que é realizada, em parte, pelo impacto nos volumes do átrio e ventrículo esquerdos, levando à disfunção sistólica e diastólica.

Para se realizarem as medidas de espessura e comprimento das cúspides, empregou-se a posição paraesternal direita longitudinal quatro câmaras, que permitiu fácil visibilização do aparato valvar, proporcionando uma medida consistente da cúspide. Boon (1998) e Pedersen et al. (1999) definiram-na como sendo a visão que fornece imagens satisfatórias do lado esquerdo do coração, tornando relativamente fácil a avaliação da valva mitral.

Outros fatores importantes na avaliação ecocardiográfica da regurgitação mitral são o tamanho e a espessura das cúspides da valva mitral. Nos seres humanos (Shah, 1994), a espessura das cúspides é um marcador de valor prognóstico, refletindo o depósito de glicosaminoglicanos nas valvas e, consequentemente, a gravidade da lesão. Ocorre a formação de nódulos com formato arredondado que se implantam sobre as bordas livres das cúspides, especialmente a septal, impedindo a coaptação adequada durante a sístole ventricular (Kogure, 1980). Esses nódulos foram constatados principalmente nos animais dos grupos II e III, estando as cúspides alongadas e espessadas (principalmente a septal). Não houve diferença no tamanho e na espessura das valvas entre os cães dos grupos I e II, embora se apresentassem anormais se comparados com os valores do grupo-controle.

Para Nakayama et al. (1996), Boon (1998) e Pedersen (2000), a dificuldade em distinguir o espessamento das cúspides entre os grupos pode ser atribuída a fatores como sobreposição e redundância das cúspides ou, ainda, aos pontos de união destas com as cordoalhas tendíneas, especialmente nas lesões discretas a moderadas. Com a progressão do quadro, a lesão torna-se mais evidente, facilitando a mensuração.

Outro fator, descrito por Nakayama et al. (1996), para verificar a limitação na obtenção dessas variáveis, é a dificuldade de avaliar o aparato valvar mitral em movimento nas frequências cardíacas elevadas. $\mathrm{O}$ emprego do cine loop nos equipamentos modernos tornou possível a observação detalhada da valva, permitindo a realização precisa das medidas. Nos animais dos grupos II e III, foi possível observar também um certo grau de espessamento nas cordoalhas tendíneas e as cúspides prolapsando para dentro do AE durante a sístole ventricular, o que pode ser considerado um fator prognóstico. Nakayama et al. (1996) relataram que a valva mitral sofre profusão em graus variáveis durante a sístole, em cães com doença mixomatosa. Segundo Jacobs et al. (1995), é comum o espessamento da cordoalha nos quadros graves de DMVM, especialmente onde se fixa à valva.

Neste estudo, observou-se que a avaliação da espessura e do tamanho das cúspides da valva mitral é uma técnica exequível na identificação de pacientes com DMVM, porém, diferente do que ocorre em seres humanos, não possibilitou a diferenciação entre pacientes portadores de insuficiência discreta e moderada. 


\section{CONCLUSÕES}

A avaliação clínica da função cardíaca possibilita classificar os cães com DMVM em três categorias, discreta, moderada e grave, de acordo com a gravidade dos sinais apresentados. O exame ecocardiográfico, modo bidimensional, utilizado na posição parasternal direita longitudinal quatro câmaras, permite a mensuração precisa do comprimento e da espessura das cúspides da valva mitral. A mensuração ecocardiográfica da valva mitral em cães com ICC possibilitou o diagnóstico da DMVM, mas não permitiu a diferenciação entre os pacientes classificados nos grupos I e II. Entretanto, mostrou-se como um importante fator de diagnóstico dos cães portadores de ICC grave (grupo III).

\section{REFERÊNCIAS BIBLIOGRÁFICAS}

ATKINS, C.E. Acquired valvular insufficiency. In: MILLER, M.S.; TILLEY, L.P. (Eds). Manual of canine and feline cardiology. 2.ed. Philadelphia: W.B. Saunders, 1995. p.129-144.

ATKINS, C.E. Evaluation of cough in dogs with mitral valve insufficiency. Comp. Cont. Educ. Pract. Vet., v.16, p.1547-1553, 1994.

BOON, J.A. Manual of veterinary echocardiography. Baltimore: Williams \& Wilkins, 1998. 478p.

BONAGURA, J.D.; BUSSADORI, C.; $\mathrm{CHURCH}, \mathrm{D}$. et al. Recommendations for the diagnosis of heart disease and the treatment of heart failure in small animals. Columbia: International Small Animal Cardiac Health Council, 1992. 32p.

BRIGHT, J.M.; MEARS, E. Chronic heart disease and its management. Vet. Clin. N. Am.: Small Anim. Pract., v.27, p.1305-1329, 1997.

CAPE, E.G.; YOGANATHAN, A.P.; LEVINE, R.A. Increased heart rate can cause underestimation of regurgitant jet size by Doppler color flow mapping. J. Am. College Cardiol., v.21, p.1029-1037, 1993.

CARABELLO, B.A. Mitral valve regurgitation. Curr. Prob. Cardiol., v.23, p.200-241, 1998.

GUGLIELMINI, C.; CHETBOUL, V.; PIETRA, $M$. et al. Influence of left atrial enlargement and body weight on the development of atrial fibrillation: retrospective study on 205 dogs. Vet. J., v.160, p.235-241, 2000.

HÄGGSTRÖM, J.; KVART, C.; HANSSON, K. Heart sounds and murmurs: changes related to severity of chronic valvular disease in the Cavalier King Charles Spaniel. J. Vet. Intern. Med., v.9, p.75-85, 1995.

HYUN, C. Mitral valve prolapse in Cavalier King Charles Spaniel: a review and case study. J. Vet. Sci., v.6, p.67-73, 2005.

JACOBS, G.J.; CALVERT, C.A.; MAHAFFEY, M.B. et al. Echocardiographic detection of flail left atrioventricular valve cusp from ruptured chordae tendineae in 4 dogs. J. Vet. Intern. Med., v.9, p.341-346, 1995.

JACOBS, G.J.; MAHJOOB, K. Influence of alterations in heart rate on echocardiographic measurements in the dog. Am. J. Vet. Res., v.49, p.548-552, 1988.

KITTLESON, M.D. Myxomatous atrioventricular valvular degeneration. In: KITTLESON, M.D.; KIENLE, R.D. (Eds). Small animal cardiovascular medicine. St. Louis: Mosby, 1998. p.297-318.

KOGURE, K. Pathology of chronic mitral valvular disease in the dog. Jpn. J. Vet. Sci., v.42, p.323-335, 1980 .

MORAIS, H.A.; PEREIRA, P.M. De onde vem este sopro? Clin. Vet., v.6, p.40-48, 2001.

MUZZI, R.A.L.; MUZZI, L.A.L.; ARAÚJO, R.B. et al. Diagnóstico ecoDopplercardiográfico da fibrose crônica da válvula mitral em cão. Arq. Bras. Med. Vet. Zootec., v.51, p.547-550, 1999.

NAKAYAMA, T.; WAKAO, Y.; NEMOTO, H. et al. Mitral valve protrusion assessed by use of B-mode echocardiography in dogs with mitral regurgitation. Am. J. Vet. Res., v.57, p.791-797, 1996.

PEDERSEN, H.D. Diagnóstico de la enfermedad de la válvula mitral mixomatosa canina. Waltham Focus, v.10, p.3-9, 2000.

PEDERSEN, H.D.; HAGGSTROM, J.; FALK, $\mathrm{T}$. et al. Auscultation in mild mitral regurgitation in dogs: observer variation, effects of physical maneuvers, and agreement with color Doppler echocardiography, and phonocardiography. $J$. Vet. Intern. Med., v.13, p.56-64, 1999. 
SHAH, P.M. Echocardiographic diagnosis of mitral valve prolapse. J. Am. Soc. Echocardiogr., v.7, p.286-293, 1994.

SISSON, D.; KVART, C.; DARKE, P.G.G. Acquired valvular heart disease in dogs and cats. In: FOX, P.R.; SISSON, D.; MOÏSE, N.S. (Eds). Textbook of canine and feline cardiology. 2.ed. Philadelphia: W.B. Saunders, 1999. p.536-565.

SISTEMA de análise estatísticas. - SAEG. Versão 7. Viçosa: UFV, 1999.

SOARES, E.C.; LARSSON, M.H.M.A.; YAMATO, R.J. Chronic valvular disease: correlation between clinical, electrocardiographic, radiographic and echocardiographic aspects in dogs. Arq. Bras. Med. Vet. Zootec., v.57, p.436-441, 2005.

STEPIEN, R.L.; BONAGURA, J.D.;
BEDNARSKI, R.M. Cardiorespiratory effects of acepromazine maleate and buprenorphine hydrochloride in clinically normal dogs. Am. J. Vet. Res., v.56, p.78-84, 1995.

TESHIMA, K.; ASANO, K.; IWANAGA, K. et al. Evaluation of left ventricular Tei Index (Index of myocardial performance) in healthy dogs and dogs with mitral regurgitation. Intern. Med., v.69, p.117-123, 2007.

THOMAS, W.P.; GABER, C.E.; JACOBS, G.J. et al. Recommendations for standards in transthoracic two-dimensional echocardiography in the dog and cat. The echocardiography Committee of the Specialty of Cardiology, American College of Veterinary Internal Medicine. J. Vet. Intern. Med., v.7, p.247-252, 1993. 